\title{
Clinical study of a CT evaluation model combined with serum CA125 in predicting the treatment of newly diagnosed advanced epithelial ovarian cancer
}

Lu Qin, Huming Huang, Mengjie Chen, Yuejuan Liang and He Wang ${ }^{*}$

\begin{abstract}
Background: The treatment of newly diagnosed advanced epithelial ovarian cancer (EOC) was predicted by an ovarian cancer computed tomography (CT) evaluation model combined with serum CA125.

Methods: Clinical data for 194 patients with advanced EOC treated with neoadjuvant chemotherapy (NACT) combined with interval debulking surgery (IDS) or primary debulking surgery (PDS) were retrospectively analyzed, and the appropriate treatment was predicted by comparing the subgroup differences in intraoperative situations, postoperative situations and survival rates.

Results: There were no significant differences with respect to operation time, intraoperative blood loss, ideal tumor cytoreductive rate or postoperative complication rate between the NACT + IDS group and the PDS group with scores less than $5($ score $<5)(p=0.764, p=0.504, p=0.906, p=0.176)$. However, there was a statistically significant difference in overall survival rate between the two groups $(p=0.029)$, with better survival in the PDS group than in the NACT + IDS group. There were significant differences between the NACT + IDS group and the PDS group with scores greater than or equal to 5 (score $\geq 5$ ). The former was better than the latter in terms of operation time, intraoperative blood loss, ideal tumor cytoreductive rate, and postoperative complication rate $(p=0.002, p=0.040$, $p=0.014, p=0.021)$. However, there was no significant difference in overall survival rate between the two groups $(p=0.383)$.
\end{abstract}

Conclusions: According to the new evaluation system, for a score $<5$, we suggest that patients with newly diagnosed advanced EOC undergo PDS; for a score $\geq 5$, we recommend NACT + IDS.

Keywords: CA125, Advanced epithelial ovarian cancer, Neoadjuvant chemotherapy, Debulking surgery

\section{Background}

Ovarian cancer is one of the most common gynecological cancers and has the highest mortality of all gynecological cancers. Since ovarian cancer has no typical clinical manifestations or exact early screening methods, Siegel et al. [1] reported that $70 \%$ of ovarian cancer patients had advanced stage disease (stage III-IV) upon presentation and that the overall 5 -year survival rate was only $27 \%$. The prognosis of advanced ovarian cancer is closely related to

\footnotetext{
* Correspondence: wanghe10430@126.com

Department of Gynecologic Oncology, Affiliated Tumor Hospital of Guangxi Medical University, 71 Hedi Road, Qingxiu District, Nanning, Guangxi 530021, People's Republic of China
}

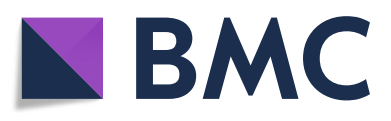

(c) The Author(s). 2018 Open Access This article is distributed under the terms of the Creative Commons Attribution 4.0 International License (http://creativecommons.org/licenses/by/4.0/), which permits unrestricted use, distribution, and reproduction in any medium, provided you give appropriate credit to the original author(s) and the source, provide a link to the Creative Commons license, and indicate if changes were made. The Creative Commons Public Domain Dedication waiver (http://creativecommons.org/publicdomain/zero/1.0/) applies to the data made available in this article, unless otherwise stated.

the volume of postoperative residual tumor. Therefore, the ability to achieve ideal tumor cytoreduction becomes a pivotal prognostic factor for advanced ovarian cancer. The ideal tumor cytoreductive surgery standard is a maximum postoperative residual tumor diameter $<1 \mathrm{~cm}$ [2]. At present, there are two primary approaches to the treatment of advanced EOC: neoadjuvant chemotherapy combined with intermittent debulking surgery (NACT + IDS) and primary debulking surgery (PDS). To predict the treatment regimen of newly diagnosed EOC patients by an ovarian cancer CT evaluation model combined with serum CA125, clinical data for 194 newly diagnosed 
advanced EOC patients who came to our hospital from March 2010 to July 2016 were retrospectively analyzed.

\section{Methods}

\section{Patient selection}

Data were collected for 194 patients who had been newly diagnosed with advanced EOC when they arrived at our hospital from March 2010 to July 2016. According to our hospital gynecologists' clinical experience, laboratory tests, imaging data and the patients' own wishes, 99 patients with advanced EOC were treated with NACT + IDS. Patients were 29 to 75 years old (mean $53.05 \pm 9.74$ years); 78 cases were in pathological stage III, and 21 were in stage IV. Preoperative chemotherapy was based on platinum-based chemotherapy, and tumor cells were destroyed by chemotherapy. These patients completed 1 2 courses of platinum-based NACT before undergoing tumor cytoreductive surgery. Ninety-five patients with advanced EOC were treated with PDS. They were 25 to 79 years old (mean $54.72 \pm 10.59$ years old) with pathological stage III (79 cases) or stage IV (16 cases) disease. According to their wishes, 176 patients underwent platinum-based systemic vein NACT, and 18 patients had no postoperative chemotherapy.

\section{Measurement of CT evaluation model and CA125}

We recorded the operation time, intraoperative blood loss, operative efficacy, postoperative recovery, postoperative complications, and postoperative follow-up of 194 patients with newly diagnosed advanced EOC. Based on the CT evaluation model of ovarian cancer patients that was established by Bristow et al. [3] in 2000, patients with a cumulative score $\geq 4$ were not suitable for PDS. According to Vorgias et al. [4], when serum CA125 levels are higher than $500 \mathrm{U} / \mathrm{ml}$, its accuracy is highest for predicting tumor cytoreductive surgery. Therefore, if at initial diagnosis the serum CA125 levels were $\geq 500 \mathrm{U} / \mathrm{ml}$, we recorded 1 point. We now establish a New Scoring System based on a CT evaluation model combined with serum CA125 for patients with newly diagnosed advanced EOC. Five points will be designated as the demarcation point. One hundred ninety-four cases were divided into 4 groups by this New Scoring System: NACT + IDS group (score $<5, n=49$ ), PDS group (score $<5, n=47$ ), NACT + IDS group (score $\geq$ $5, n=50$ ) and PDS group (score $\geq 5, n=48$ ). In accordance with the intraoperative situation, postoperative situation and prognosis of patients in each group, appropriate treatment was predicted. The size of the residual tumor after surgery is an important prognostic factor. According to the 2014 National Comprehensive Cancer Network (NCCN) guidelines for ovarian cancer, non-ideal tumor cytoreductive surgery is indicated by postoperative residual ovarian cancer lesions $\geq 1 \mathrm{~cm}$ [5]. Four groups of patients were followed up until April 2017 (Table 1).
Table 1 New Scoring System based on a CT evaluation model combined with serum CA125

\begin{tabular}{ll}
\hline The initial diagnosis of serum CA125 levels $(\mathrm{U} / \mathrm{ml})$ & Point value \\
\hline$\geq 500$ & 1 \\
$<500$ & 0 \\
Predictive index parameter & Point value \\
Peritoneal thickening & 2 \\
Peritoneal implants $\geq 2 \mathrm{~cm}$ & 2 \\
Small bowel mesentery disease $\geq 2 \mathrm{~cm}$ & 2 \\
Large bowel mesentery disease $\geq 2 \mathrm{~cm}$ & 2 \\
Omentum extension to stomach, spleen, or lesser sac & 2 \\
Extension to pelvic sidewall, parametria, or hydroureter & 2 \\
Ascites-large volume (seen on all cuts) & 2 \\
Performance status $\geq 2$ & 2 \\
Suprarenal paraaortic lymph nodes $\geq 1 \mathrm{~cm}$ & 2 \\
Diaphragm or lung base disease $\geq 2 \mathrm{~cm}$ or confluent & 1 \\
plaque & \\
Inguinal canal disease or lymph nodes $\geq 2 \mathrm{~cm}$ & 1 \\
Liver lesion $\geq 2 \mathrm{~cm}$ on surface or any size parenchymal & 1 \\
lesion & \\
Porta hepatis or gallbladder fossa disease $\geq 1$ cm & 1 \\
Infrarenal paraaortic lymph nodes $\geq 2 \mathrm{~cm}$ & 1 \\
\hline
\end{tabular}

\section{Statistical analysis}

SPSS 20.0 software was used for statistical analysis. Normally distributed data are presented as the mean \pm standard deviation $(\mathrm{x} \pm \mathrm{S})$. Skewed data are presented as $\mathrm{M}\left(\mathrm{P}_{25} \sim \mathrm{P}_{75}\right)$. The data were analyzed by $t$-test, $\mathrm{X}^{2}$ test, non-parametric test or survival analysis. For all comparisons, differences were considered significant when $p<0.05$. All findings were confirmed in at least one additional independent experiment.

\section{Results}

\section{Clinicopathological factors in patients}

After statistical analysis, patients in the NACT + IDS group (score $<5$ ) were $53.16 \pm 9.40$ years old, and patients in the PDS group (score $<5$ ) were $53.64 \pm 10.79$ years old. There was no significant difference in age between the two groups $(p=0.818)$. There was also no significant difference in clinical stage, pathological type or histological grade between the two groups ( $p=0.343, p=0.970, p=0.598$ ). Patients in the NACT + IDS group (score $\geq 5$ ) were $52.94 \pm 10.16$ years old, and patients in the PDS group (score $\geq 5$ ) group were $55.77 \pm 10.39$ years old. There was no significant difference in age between the two groups $(p=0.176)$. There was also no significant difference in clinical stage, pathological type or histological grade between the two groups $(p=0.876, p=0.819, \quad p=0.448)$ (Table 2). 
Table 2 Clinicopathological factors in the NACT + IDS and PDS groups of patients grouped according to the New Scoring System

\begin{tabular}{|c|c|c|c|c|c|c|}
\hline \multirow{2}{*}{$\begin{array}{l}\text { Clinicopathological } \\
\text { factors }\end{array}$} & \multicolumn{3}{|c|}{ Groups with score $<5$} & \multicolumn{3}{|c|}{ Groups with score $\geq 5$} \\
\hline & NACT + IDS & PDS & $p$ & $N A C T+$ IDS & PDS & $p$ \\
\hline Age (y) & $(53.16 \pm 9.40)$ & $(53.64 \pm 10.79)$ & 0.818 & $(52.94 \pm 10.16)$ & $(55.77 \pm 10.39)$ & 0.176 \\
\hline \multicolumn{7}{|l|}{ Stage } \\
\hline III & 38 & 40 & 0.343 & 40 & 39 & 0.876 \\
\hline IV & 11 & 7 & & 10 & 9 & \\
\hline \multicolumn{7}{|l|}{ Histology } \\
\hline Serous & 42 & 41 & 0.970 & 44 & 41 & 0.819 \\
\hline Mucous & 6 & 5 & & 5 & 5 & \\
\hline Endometrioid & 1 & 1 & & 1 & 2 & \\
\hline \multicolumn{7}{|l|}{ Grade } \\
\hline G1 & 15 & 19 & 0.598 & 16 & 19 & 0.448 \\
\hline G2 & 4 & 3 & & 3 & 5 & \\
\hline G3 & 30 & 25 & & 31 & 24 & \\
\hline
\end{tabular}

$N A C T+I D S$ neoadjuvant chemotherapy and interval debulking surgery, $P D S$ primary debulking surgery

\section{Comparison of intraoperative and postoperative} situations in the NACT + IDS and PDS groups (score $<5$ ) There were no significant differences in terms of operation time, intraoperative blood loss or ideal tumor cytoreductive rate between the two groups $(p=0.764, p=0.504, p=$ 0.906). The incidence of complications in the NACT + IDS group was $12.24 \%$, and that in the PDS group was $14.89 \%$. There was no significant difference in the incidence of complications between the two groups after $x^{2}$ tests $(p=$ 0.705). On follow-up of the NACT + IDS group, 20 patients survived, 24 died, and 5 were lost to follow-up. On follow-up of the PDS group, 28 patients survived, 12 died, and 7 were lost to follow-up. There was a significant difference in overall survival between the two groups $(p=0.029)$ (Table 3, Fig. 1).

\section{Comparison of intraoperative and postoperative} situations in the NACT + IDS and PDS groups (score $\geq 5$ ) There were significant differences in operation time, intraoperative blood loss and ideal tumor cytoreductive rate between the two groups ( $p=0.002, p=0.040, p=0.014$ ). That is, the operation time and intraoperative blood loss of patients in the NACT + IDS group were lower than those of patients in the PDS group. In addition, the ideal rate of reduction in NACT + IDS patients was higher than that it was in PDS patients. The incidence of complications was
$4.00 \%$ in the NACT + IDS group and $18.75 \%$ in the PDS group. There was an obvious and significant difference in the incidence of complications between the two groups after $\chi^{2}$ tests $(p=0.021)$. We can conclude that the incidence of complications was lower in the NACT + IDS group than in the PDS group. On follow-up of the NACT + IDS group, 20 patients survived, 24 died, and 5 were lost to follow-up. On follow-up of the PDS group, 28 patients survived, 12 died, and 7 were lost to follow-up. There was no significant difference in overall survival between the two groups ( $p=0.383$ ) (Table 4, Fig. 2).

\section{Comparative analysis of ideal tumor reduction at} operation in the two PDS groups with scores $<5$ and $\geq 5$

According to case information, the two groups of patients with scores $<5$ and $\geq 5$ in terms of ideal tumor reduction were significantly different macroscopically. However, after statistical analysis, there was no statistically significant difference between the two groups of patients in terms of ideal tumor reduction rate $(p=0.296)$.

\section{Discussion}

Ovarian cancer not only is a serious threat to the health and life of women but also has the highest mortality rate of all gynecological cancers. Because there is no exact method of screening, $70 \%$ of patients have reached the

Table 3 Surgery parameters in the NACT + IDS and PDS groups of patients with score $<5$

\begin{tabular}{llll}
\hline Index & NACT + IDS & PDS & \\
\hline Number of cases & 49 & 47 & $/$ \\
Operation time (min) & $285.04 \pm 92.24$ & $278.57 \pm 116.32$ & 0.764 \\
Intraoperative blood loss (ml) & $550(340 \sim 1300)$ & $700(300 \sim 1200)$ & 0.504 \\
Ideal tumor cytoreductive surgery & 37 & 35 & $/$ \\
Ideal tumor cytoreductive rate (\%) & 75.51 & 74.47 & 0.906 \\
\hline
\end{tabular}




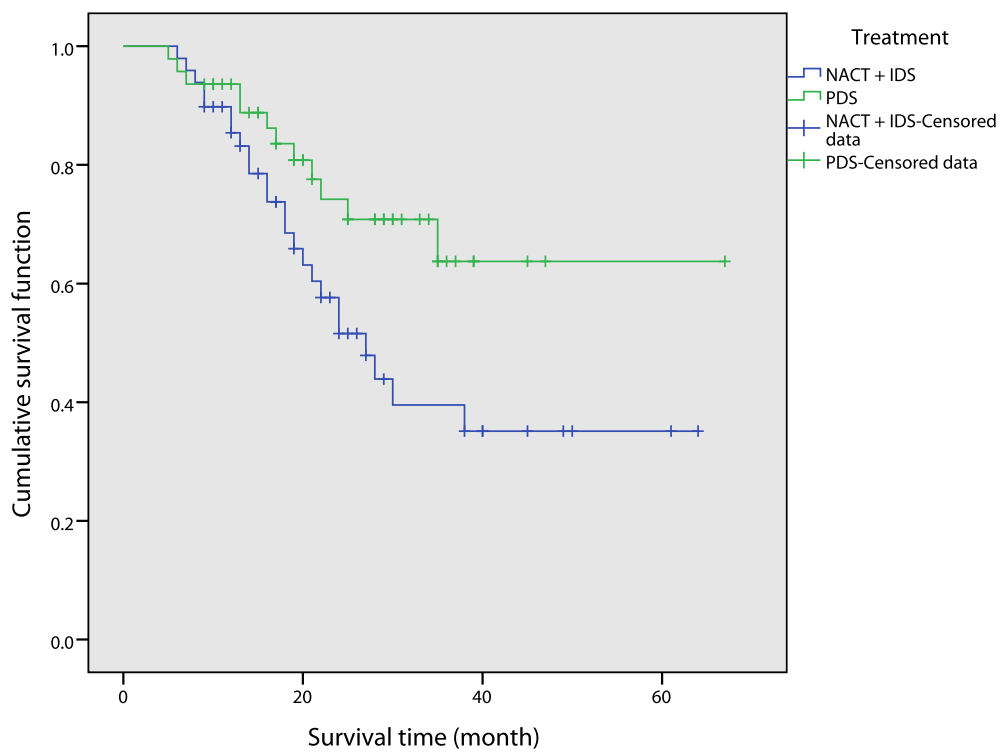

Fig. 1 Overall survival rates were compared between the NACT + IDS and PDS groups with a score $<5$

advanced stage by the time of presentation and often have abdominal metastasis, organ metastasis, ascites and pleural effusion. At present, the treatment of newly diagnosed patients with advanced epithelial ovarian cancer (EOC) is divided into two types: neoadjuvant chemotherapy combined with interval debulking surgery (NACT + IDS) and primary debulking surgery (PDS). NACT, also known as primary chemotherapy, refers to systemic chemotherapy that is administered before local treatment (such as radiotherapy or surgery). This treatment is primarily administered when PDS cannot achieve ideal tumor cytoreduction and/or patients are in poor condition and cannot tolerate tumor cytoreductive surgery. The ideal tumor cytoreductive rate can be improved by NACT [6]. Ovarian cancer is very sensitive to chemotherapy drugs. About 70 to $80 \%$ of patients with ovarian cancer have a complete response after chemotherapy [7]. Studies have shown that NACT has the following advantages: it kills metastastic cells surrounding the lesion, loosens the adhesion of the tumor and surrounding tissue, reduces tumor volume, improves the ideal tumor cytoreductive rate, and reduces the difficulty and complications of surgery [8]; it controls ascites and pleural effusion to improve the overall condition of patients and improve their surgical tolerance; it shortens the operation time, reduces intraoperative blood loss, and improves patient quality of life [9]; and it puts the tumor cells into a "dormant state", reducing tumor spread and tumor cell seeding by surgery due to intraoperative squeezing and mechanical trauma stimulation, thereby reducing recurrence. However, the use of NACT for the treatment of patients with advanced ovarian cancer has been continuously controversial. For patients with advanced ovarian cancer who remain in good condition, studies have shown that PDS can achieve better tumor reduction and prognosis than NACT.

Based on the CT evaluation model of ovarian cancer patients that was established by Bristow et al. in 2000, patients with a cumulative score $\geq 4$ (score $\geq 4$ ) are not suitable for PDS. They noted that such patients should undergo NACT until the condition is under control prior to surgery. In addition, a large number of studies have shown that more than $85 \%$ of patients with ovarian cancer have a serum CA125 level higher than $35 \mathrm{U} / \mathrm{mL}$, and serum CA125 levels are closely related to disease status in $93 \%$ of patients. Therefore, the level of serum CA125 is considered strong

Table 4 Surgery parameters in the NACT + IDS and PDS groups of patients with score $\geq 5$

\begin{tabular}{llll}
\hline Index & NACT + IDS & PDS & \\
\hline Number of cases & 50 & 48 & $/$ \\
Operation time (min) & $270.64 \pm 92.64$ & $339.48 \pm 119.04$ & 0.002 \\
Intraoperative blood loss (ml) & $550(275 \sim 1100)$ & $950(400 \sim 1675)$ & 0.040 \\
Ideal tumor cytoreductive surgery & 43 & 31 & $/$ \\
Ideal tumor cytoreductive rate (\%) & 86.00 & 64.58 & 0.014 \\
\hline
\end{tabular}




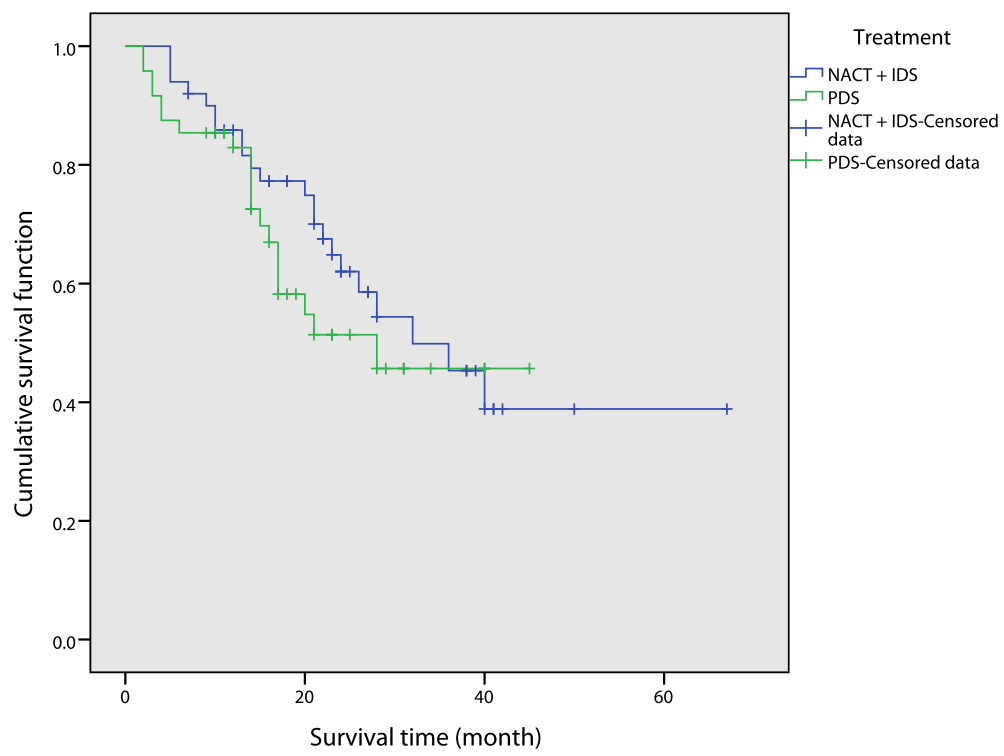

Fig. 2 Overall survival rates were compared between the NACT + IDS and PDS groups with a score $\geq 5$

evidence in the evaluation of the ability of patients to achieve ideal tumor cytoreduction [10]. Vorgias et al. found that when serum CA125 levels were higher than $500 \mathrm{U} / \mathrm{ml}$, the accuracy of its predictive ability was highest for patients undergoing tumor cytoreductive surgery to achieve the ideal standard, accounting for $84.2 \%$ in all observed ideal tumor cytoreductive surgeries. Obeidat et al. [11] supported this view and reported that a preoperative serum CA125 level of $500 \mathrm{U} / \mathrm{ml}$ was the most valuable indicator of whether ideal tumor cytoreductive surgery could be achieved. This indicator's sensitivity and specificity were 72 and $73 \%$, respectively. The positive predictive value was $68 \%$. However, some investigators believed that the negative predictive value of CA125 is poor and that this measure cannot be used independently as an index to predict surgery. Therefore, in order to predict more suitable treatment for patients, we have assessed patients with newly diagnosed advanced EOC by taking advantage of an ovarian cancer CT evaluation model established by Bristow et al. combined with serum CA125 levels.

By comparing two groups (NACT + IDS and PDS), we found that there was no significant difference in the operation time, intraoperative blood loss, the ideal tumor cytoreductive rate or postoperative complications between the two groups of patients with a score $<5(p=0.764, p=0.504, p=0.906, p=0.705)$. However, the overall survival rate was significantly different between the two groups $(p=0.029)$, with the PDS group having superior survival to the NACT + IDS group. This finding suggests that PDS did not show any advantage in terms of intraoperative and postoperative complications compared with the NACT + IDS group for patients with newly diagnosed advanced EOC when the score was $<5$, but the postoperative quality of life and long-term prognosis of these patients were significantly better than those of the NACT + IDS group. For scores $<5$, there was no significant difference between the NACT + IDS and PDS groups in terms of operation time, intraoperative blood loss and ideal tumor cytoreduction. Therefore, in order to avoid side effects and adverse reactions brought on by unnecessary chemotherapy, as well as excessive treatment costs and lengths of treatment that may delay the optimal operation time, we support the notion that advanced EOC patients should undergo PDS. In the two groups of patients with a score $\geq 5$, the operation time of the NACT + IDS group was significantly shorter than that of the PDS group $(p=0.002)$. The amount of blood loss during operation in the former was less than that in the latter, and the ideal cytoreductive rate was higher $(p=0.040, p=0.014)$. This finding showed that for scores $\geq 5$, patients undergoing NACT could significantly improve their condition prior to surgery to reduce surgical difficulty and risks. In addition, the incidence of postoperative complications in the NACT + IDS group was significantly lower than that in the PDS group $(p=0.021)$. This result suggested that NACT could significantly reduce the incidence of complications and improve the quality of life of patients who were newly diagnosed with severe illness. None of these patients could undergo PDS immediately. In terms of overall survival rate, we found no significant difference between the two groups of patients $(p=0.383)$. NACT + IDS did not appear to improve prognosis. This result was in line with that of many published reports. Lozzi et al. [12] 
conducted a paired study of neoadjuvant chemotherapy with patients treated with standard treatment over the same period and showed no significant differences between the neoadjuvant chemotherapy group and the standard treatment group in terms of median survival time, median progression-free survival time and 3-year survival rate. Wright et al. [13] compared the efficacy of PDS and NACT + IDS in elderly patients with ovarian cancer using SEER data. They noted that the average survival of patients in the NACT + IDS and PDS groups was 15.8 months and 28.8 months, respectively, with 2 -year survival rates of 36 and $56 \%$, respectively. Fago-Olsen et al. [14] reported that the average overall survival of patients without residual tumor in the PDS group was better than that of those in the NACT + IDS group, at 55.5 and 36.7 months, respectively $(p=0.002)$. Simultaneously, patients in the NACT + IDS group had an increased risk of death after 2 years. We speculated that the reason for this analysis may be as follows: although NACT can reduce the difficulty and risk of surgery, the criterion for satisfactory tumor shrinkage in these studies was the same for patients with intermittent cytoreductive surgery following neoadjuvant chemotherapy as for direct cytoreductive surgery, namely, residual lesions less than $1 \mathrm{~cm}$. The number of chemotherapy regimens after intermittent neoplastic cytoreductive surgery following neoadjuvant chemotherapy is fewer than the number after direct surgery, because neoadjuvant chemotherapy may increase the chemoresistance potential of ovarian cancer cells. However, NACT + IDS did not improve patient prognosis. By analyzing the ideal tumor cytoreductive rates of two PDS groups of patients who scored $<5$ and $\geq 5$, the difference in the ideal tumor cytoreductive rate of the two groups was approximately $10 \%$, and the ideal surgical cytoreductive rate in patients in the PDS group and with a score $<5$ was significantly higher than that of patients with a score $\geq 5$. Although there was no statistically significant difference between the two groups $(p=0.296)$, we cannot conclude that there was no difference in the rate of reduction between the two groups. We believe the reason may stem from limitations in the collection of cases. For the treatment of patients with newly diagnosed advanced EOC, the choice of the re-established New Scoring System score cut-off point has substantial guiding significance. Whether there is a score $<5$ or $\geq 5$, patients with ovarian cancer can choose a relatively more favorable treatment. Therefore, we believe the choice of the cut-off point was reasonable. Taken together, we hypothesize that patients with newly diagnosed advanced EOC and score $<5$ who can tolerate PDS at presentation may be better able to manage the optimal surgical treatment time, shorten the duration of patient treatment, reduce pain, reduce treatment costs and improve long-term outcomes. However, patients with a score $\geq$ 5 who were treated with NACT + IDS were more conducive to reducing the difficulty and risk of surgery, improving the reduction rate of tumor cells and improving the quality of life.

\section{Conclusion}

Ovarian cancer has the highest mortality rate of all gynecological tumors. Most patients are already in advanced stages when they present for care. Advanced ovarian cancer tumor cells are widely disseminated. Their lesions are large and heavily infiltrate surrounding tissue. They also suppress organ function. Relevant reports show that only $42 \%$ of patients with severe disease can achieve ideal tumor reduction [15]. To delay the progression of the disease and to improve the quality of life, it is very important to choose treatment modalities for patients with newly diagnosed advanced epithelial ovarian cancer (EOC). We have evaluated the treatment by establishing a new evaluation system (new ovarian cancer CT model combined with serum CA125 levels). When patients are newly diagnosed with advanced EOC and have a score $<5$, we suggest primary debulking surgery because this approach is able to save treatment time and costs and to improve the long-term prognosis. When the score is greater than or equal to 5 points, we are more inclined to recommend NACT + IDS in order to reduce the difficulty and risk of surgery and to improve the quality of life. There remains space for improvement and refinement of treatment options for patients with newly diagnosed EOC under the New Scoring System. Although at this stage of our research we can only provide some treatment ideas, we have laid a solid foundation for future research and analysis. There remain statistics from studies in other countries that show that NACT failed to improve 5-year survival rate and median survival time in patients with advanced ovarian cancer [16]. Therefore, in the future, we also need to explore and verify the treatment modalities of NACT and to conduct additional research and analysis on the New Scoring System to further demonstrate the rationale and validity of the scoring system and to provide strong evidence to support the choice of treatment modality.

\footnotetext{
Abbreviations

EOC: Epithelial ovarian cancer; IDS: Interval debulking surgery; NACT: Neoadjuvant chemotherapy; PDS: Primary debulking surgery

Acknowledgements

Thanks for the corresponding author's guidance and other authors' assistance.

Funding

This study was funded by a special project on the prevention and control of major chronic noncommunicable diseases in Chongqing, China: a research project on ovarian cancer treatment programs and clinical pathway planning. The funding item number is 2016YFC1303702.
} 


\section{Availability of data and materials}

The data are not anonymized and kept at the institute curie. They may be provided, where necessary, by the corresponding author.

\section{Authors' contributions}

LQ made substantial contributions to conception and design, planned and ran the experiments, collected data, performed data analysis, interpreted the results, reviewed the literature and wrote the manuscript. $\mathrm{HH}$ contributed to data collection. MC contributed to the literature discussion and conclusion. YL supervised the statistical analysis. HW gave final approval of the version to be published. All authors read and approved the final manuscript.

\section{Ethics approval and consent to participate}

This study did not involve human and animal experiments.

\section{Competing interests}

The authors declare that they have no competing interests.

\section{Publisher's Note}

Springer Nature remains neutral with regard to jurisdictional claims in published maps and institutional affiliations.

Received: 9 March 2018 Accepted: 23 May 2018

Published online: 18 June 2018

\section{References}

1. Siegel R, Ma J, Zou Z. Cancer statistics,2014. Cancer. 2014;64(1):9-29.

2. Ce B, Xia Z. Neoadjuvant chemotherapy in the treatment of advanced ovarian cancer. J Pract Obstet Gynecol. 2015;04:263-6.

3. Bristow RE, Duska LR, Lambrou NC, et al. A model for predicting surgical outcome in patients with advanced ovarian carcinoma using computed tomography. Cancer. 2000;89(7):1532-40.

4. Vorgias G, lavazzo C, Savvopoulos P, et al. Can the preoperative ca-125 level predict optimal cytoreduction in patients with advanced ovarian carcinoma?A single institution cohort study. Gynecol Oncol. 2009;112(1):11-5.

5. Colombo N, Van Gorp T, Parma G, et al. Ovarian cancer. Oncol Hematol. 2006:60(2):159-79.

6. Kang S. Does neoadjuvant chemotherapy increase optimal cytoreduction rate in advanced ovarian cancer? Meta-analysis of 21 studies. Surg Oncol. 2009;16(8):2315-20.

7. McGuire WP, Hoskins WJ, Brady MF, et al. Cyclophosphamide and cisplatin compared with paclitaxel and cisplatin in patients with stage III and stage IV ovarian cancer. N Engl J Med. 1996;334(1):1-6.

8. Xuemei J, Huihua D, Shuyu W, Wei X, Zhihong Z. The effect of neoadjuvant chemotherapy in gynecologic malignancies. Jiangsu Med. 2010;09:1017-20.

9. Tongwei R. Application of neoadjuvant chemotherapy in the treatment of advanced ovarian cancer. Chinese and Foreign Medical. 2009;22:8-10.

10. Ying Z, Yingli Z, Ping Z. Progress in Neoadjuvant Chemotherapy for Advanced Ovarian Cancer. J Oncol. 2014;11:942-6.

11. Obeidat B, Latimer J. Can optimal primary cytoreduction be predicted in advanced stage epithelial ovarian cancer? Role of preoperative serum CA-125 level. Gynecol Obstet Investig. 2004:57(3):153-6.

12. Loizzi V, Cormio G, Resta L, et al. Neoadjuvant chemotherapy in advanced ovarian cancer:a case-control study[J]. Int J Gynecol Cancer. 2005;15(2):217-23.

13. Wright JD, Ananth CV, Tsui J, et al. Comparative effectiveness of upfront treatment strategies in elderly women with ovarian cancer[]]. Cancer. 2014;120(8):1246-54.

14. Fago-Olsen $\mathrm{CL}$, Ottesen $\mathrm{B}$, Kehlet $\mathrm{H}$, et al. Dose neoadjuvant chemotherapy impair long-term survival for ovarian cancer patients?A nationwide Danish study[]]. Gynecol Oncol. 2014;132(2):292-8.

15. Bristow RE, Tomacruz RS, Armstrong DK, et al. Survival effect of maximal cytoreductive surgery for advanced ovarian carcinoma during the platinum era: a meta-analysis. J Clin Oncol. 2002;20(5):1248-59.

16. Schwart PE. Contemporary considerations for neoadjuvant chemotherapy in primary ovarian cancer[]].Gynecology. Cancers. 2009;11(6):457-65.

\section{Ready to submit your research? Choose BMC and benefit from:}

- fast, convenient online submission

- thorough peer review by experienced researchers in your field

- rapid publication on acceptance

- support for research data, including large and complex data types

- gold Open Access which fosters wider collaboration and increased citations

- maximum visibility for your research: over $100 \mathrm{M}$ website views per year

At BMC, research is always in progress.

Learn more biomedcentral.com/submissions 